\title{
Fatal and mild primary dengue virus infections imported to Norway from Africa and south-east Asia, 2008-2010
}

K Vainio ${ }^{1}$, S Noraas ${ }^{2}$, M Holmberg ${ }^{3}$, H Fremstad ${ }^{1}$, M Wahlstrøm ${ }^{4}$, G Ånestad ${ }^{1}$, S Dudman (susannegjeruldsen.dudman@fhi.no) ${ }^{1}$

1. Norwegian Institute of Public Health, Department of Virology, Oslo, Norway

2. Sørlandet Hospital, Kristiansand, Norway

3. Vestfold Hospital, Tønsberg, Norway

4. Swedish Institute for Infectious Disease Control, Solna, Sweden

Citation style for this article:

Vainio K, Noraas S, Holmberg M, Fremstad H, Wahlstrøm M, Ånestad G, Dudman S. Fatal and mild primary dengue virus infections imported to Norway from Africa and south-east Asia, 2008-2010. Euro Surveill. 2010;15(38):pii=19666. Available online: http://www.eurosurveillance.org/ViewArticle.aspx?Articleld=19666

Article published on 23 September 2010

Between 2008 and 2010, eight cases of viraemic dengue fever in travellers were diagnosed in Norway. They had returned from Eritrea, Thailand and Indonesia. All cases were primary dengue infections, seven noncomplicated dengue fever and one dengue shock syndrome with a fatal outcome. Four patients were infected with dengue virus serotype 1 , one with type 2 and three with type 3. Two cases from Thailand, the fatal case and the two imported from Eritrea were infected with type 1.

\section{Introduction}

Global incidence of dengue fever has increased strongly in recent decades, and dengue infections are now endemic in more than 120 countries throughout the world [1-3]. South-east Asia is the most important region of origin for the import of dengue fever into Europe [4]. In recent years, dengue virus has become a more prevalent cause of imported fever in Norwegian patients than malaria. Due to this increase, the Norwegian Institute of Public Health (NIPH) has recently proposed to the health authorities to make dengue fever a notifiable disease. Most cases diagnosed in Norway have been mild, but there have also been several cases with complicated dengue infections, including one fatal case in 2005 [5].

Of all dengue cases confirmed at the NIPH, we describe here the eight viraemic cases imported to Norway between 2008 and 2010.

\section{Case descriptions}

The eight viraemic cases reported in Norway between $2008(n=1), 2009(n=1)$ and $2010(n=6)$, were imported from Eritrea $(n=2)$, Thailand $(n=4)$ and Indonesia $(n=2)$. The patient's ages ranged from 19 to 65 years, five females and three males. None of the cases had evidence of previous dengue virus infection based on their medical history and serological evidence. Seven of the cases had non-complicated dengue fever, but one patient suffered from dengue shock syndrome with a fatal outcome.

The fatal case first presented to the local health centre with a febrile viral influenza-like illness four days after returning from Thailand [6]. Nine days after returning, the patient visited the emergency centre as no relief was obtained from using paracetamol and ibuprofen, but returned home to continue ibuprofen treatment. Twelve hours later the patient was admitted to the intensive care unit, but was then suffering from circulatory collapse and died within a few hours. During the resuscitation attempts there was abnormal bleeding from the endotracheal tube and needle injection sites. Laboratory results showed a fall in haemoglobin from 15 to $7 \mathrm{~g} / \mathrm{dL}$ and thrombocytopenia.

Another patient returning with dengue fever from Bali was examined for airway infections due to hoarseness and nasal congestion. Mycoplasma pneumoniae was detected by PCR in nasopharyngeal secretions and erythromycin tablets were prescribed. Clinical characteristics of all patients and their laboratory results are displayed in the Table.

\section{Laboratory methods}

Acute phase sera were obtained from the eight patients. Cases with no previous history of dengue virus infection and acute serum negative for anti-dengue IgG were defined as primary infections. Convalescent sera were available from only three patients and were taken 18-22 days after the acute sera. The acute samples were initially tested at the local laboratory and the positive samples were then referred to the virology laboratory at the NIPH for confirmation, except for one sample which was analysed directly at the NIPH. Infection with dengue virus was initially diagnosed in seven of the travellers by Panbio Dengue Duo IgM and IgG Rapid Strip Test (Inverness Medical Innovations, Australia) or SD Bioline Dengue NS1 Antigen and IgG/ IgM tests (Standard Diagnostics, South Korea). All 


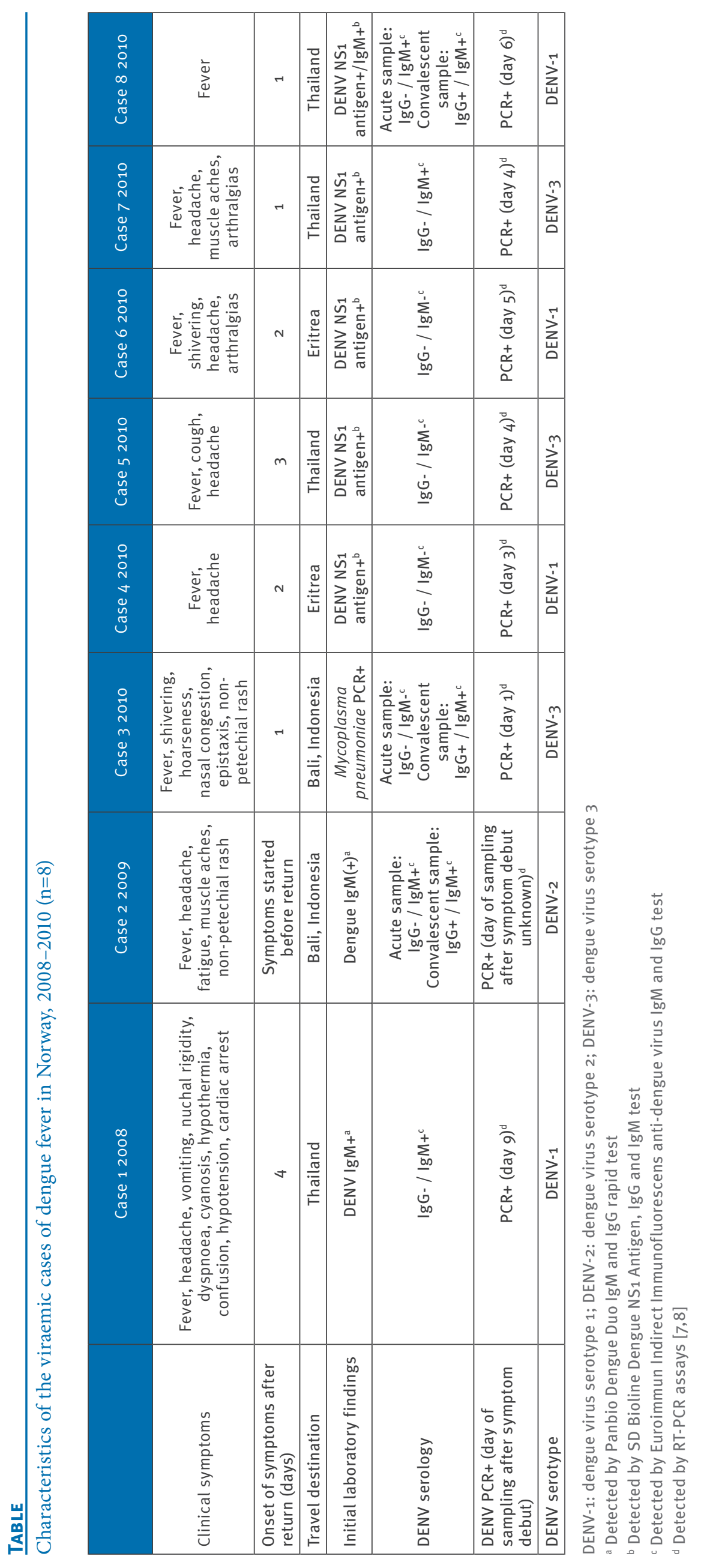


samples were further analysed at the NIPH for the presence of dengue IgG and IgM antibodies using a commercial indirect immunofluorescence assay (IFA) (Euroimmun AG, Germany) and by a reverse transcription (RT) PCR detecting the four dengue serotypes $[7,8]$. The virus strains were characterised by direct sequencing of the PCR-products and a phylogenetic tree was obtained by comparing these strains with other dengue virus strains available in the NCBI GenBank sequence database.

\section{Discussion and conclusion}

We have described eight cases of viraemic dengue virus primary infection imported from endemic areas to Norway in 2008 to 2010 , one of them with a fatal outcome. All patients developed fever within four days after returning to Norway, and the serological analyses demonstrated that they suffered from primary dengue infections. Anti-dengue IgM antibodies were detected in acute samples from the fatal case, in addition to the dengue virus serotype 2-positive case from 2009 and two cases from 2010. During primary infection, IgM and IgG antibodies are usually detectable from respectively five and 14 days after onset of symptoms

\section{FIGURE}

Phylogenetic tree comparing published dengue viruses sequences with those from viruses isolated in Norway, 2008-2010 $(\mathrm{n}=8)$

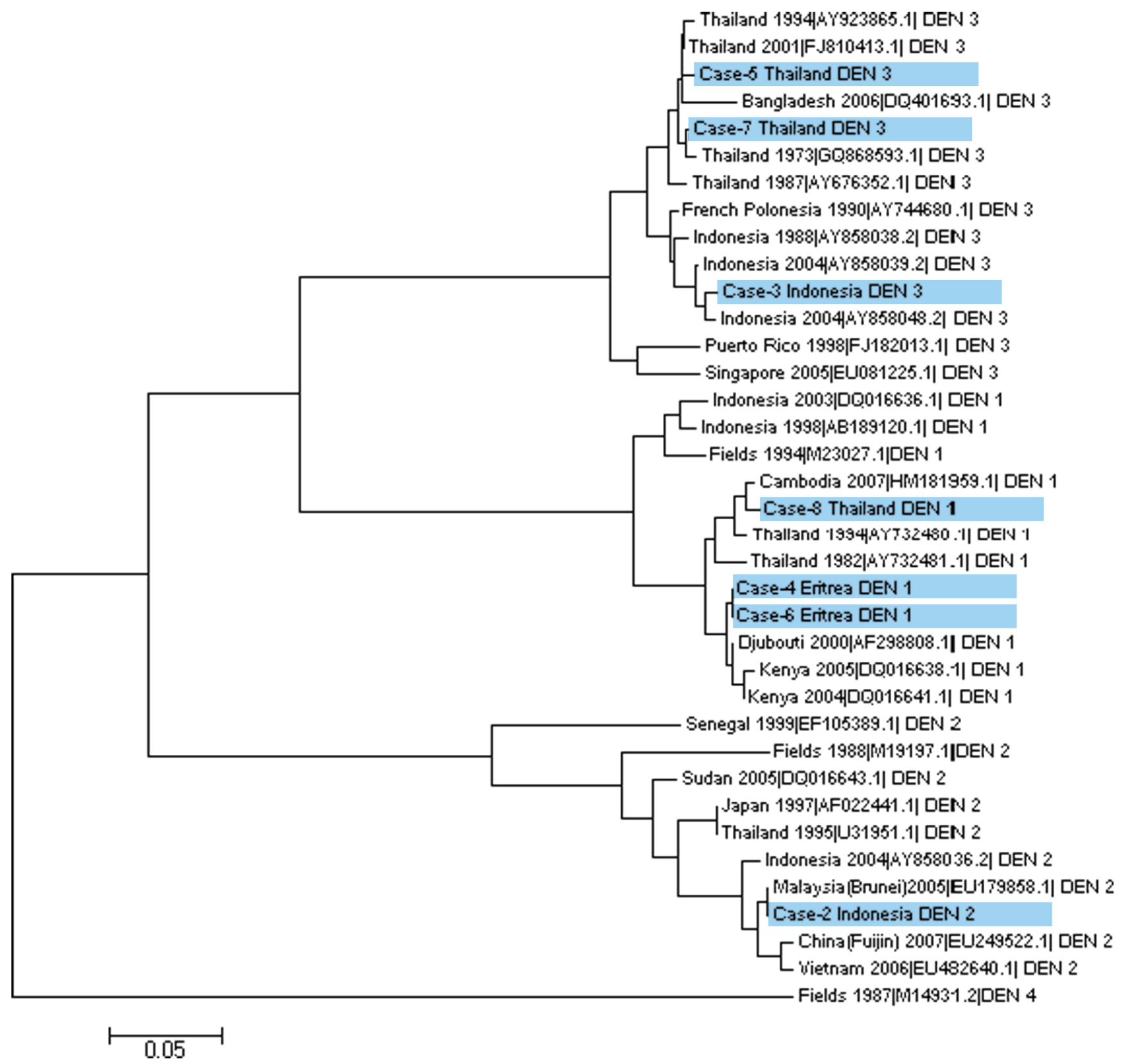

The tree is based on an approximately $300 \mathrm{nt}$ fragment of the E glycoprotein gene. Sequence identification of selected dengue virus sequences is as follows: country of origin, year of isolation and NCBI GenBank accession number. The sequences determined in our study are identified by case number and country of origin. Phylogenetic analysis was conducted using MEGA 4 [12], and the tree was constructed using neighbour-joining method. 
$[3,9]$. The fatal case highlights that fatal or severe dengue fever can also be caused by a primary infection. Severe or fatal dengue fever cases are more frequent in secondary than primary infections, but fatal primary dengue virus infection has been described in earlier reports $[3,5,9]$.

For the reported cases, the rapid NS1 antigen tests were helpful for the initial diagnosis of dengue fever in the early phase of the disease. Our results show that dengue virus can be detected by NS1 antigen tests in patients who are negative for anti-dengue $\operatorname{Ig} G$ and IgM.

This report shows the importance of performing dengue virus diagnostics in febrile patients returning from endemic areas even if other pathogens have been detected. Awareness of the different causes of travelrelated infections and early inclusion of these in the differential diagnosis is particularly important in the context of destinations with a risk of such transmission.

Other studies have shown that most dengue virus infections diagnosed in European countries have been imported from Asia or the Americas, and in these regions all four dengue virus types have been shown to circulate $[9,10]$. Six of our study cases had returned from south-east Asia with dengue virus infection caused by virus serotype 1,2 or 3 . Import of dengue virus serotype 4 into Norway has so far not been reported.

Two of the cases in this study were imported from Eritrea, where only one dengue virus serotype 3 isolate has been reported earlier [10]. This country is not a specifically popular destination for Norwegian travellers and we are not aware of a concurrent outbreak in Eritrea. To date, there have been few reports of viraemic dengue fever cases imported into Europe from Africa. This may be due to underreporting in some African countries, as well as lack of adequate diagnostic tools [11]. Dengue surveillance is poorly implemented in Africa and surveillance of febrile travellers returning to Europe will add new knowledge on dengue virus distribution throughout Africa.

A comparison of sequences obtained in this study and from studies published elsewhere, are shown in the phylogenetic tree (Figure). In general, the sequence similarity between isolates of one dengue serovirus type was greater than $95 \%$. The dengue virus serotype 1 isolates from Eritrea were closely related to dengue virus serotype 1 isolated in Kenya in 2004-5. Similarly, the dengue virus serotypes 1,2 and 3 imported from south-east Asia in our study clustered together with the respective serotypes reported from this area earlier. To our knowledge, only few reports of dengue virus serotype 1 isolates from East Africa have been published [10], and this study provides evidence that this serotype 1 is circulating in this area.
Our report confirms that returning travellers may serve as sentinels for local outbreaks of dengue fever in endemic areas. The worldwide surveillance of dengue virus requires simple and accurate methods for the identification of virus types and is especially important since air travellers move quickly between endemic and non-endemic regions, allowing introductions of dengue virus to new areas that already are populated with Aedes mosquitoes.

\section{References}

1. Kyle JL, Harris E. Global spread and persistence of dengue. Annu Rev Microbiol. 2008;62:71-92.

2. World Health Organization. Dengue and dengue haemorrhagic fever. Fact sheet $N^{\circ} 117$. Geneva: World Health Organization; May 2008. Available from: http://www.who.int/mediacentre/ factsheets/fs117/en/

3. Halstead SB. Dengue. Lancet. 2007;370(9599):1644-52.

4. Jelinek T. Trends in the epidemiology of dengue fever and their relevance for importation to Europe. Euro Surveill. 2009;14(25):pii=19250. Available from: http://www. eurosurveillance.org/ViewArticle.aspx?Articleld=19250

5. Jensenius M. Berild D. Ormaasen V. Maehlen J. Lindegren G. Falk KI. Fatal subarachnoidal haemorrhage in a Norwegian traveller with dengue virus infection. Scand J Infect Dis. 2007;39(3):272-4.

6. Blystad H, Borgen K. Dødelig denguevirus-infeksjon etter turistopphold i Thailand. [Fatal dengue virus infection after tourist visit to Thailand]. MSIS-rapport 2008;9. [Accessed 29 August 2010]. Oslo: Nasjonalt folkehelseinstitutt; 30 April 2008. Available from: http://www.fhi.no/dav/6c111c7181.pdf

7. Lindegren G, Vene S, Lundkvist A, Falk KI. Optimized Diagnosis of Acute Dengue Fever in Swedish Travelers by a Combination of Reverse Transcription-PCR and Immunoglobulin M Detection. J Clin Microbiol. 43(6):2850-5, 2005

8. Domingo C, Palacios G, Niedrig M, Cabrerizo $M$, Jabado 0 , Reyes N, et al. A New Tool for the Diagnosis and Molecular Surveillance of Dengue Infections in Clinical Samples. Dengue Bulletin. 2004;28. New Delhi: WHO Regional Office for SouthEast Asia; 2010. Available from: http://www.searo.who.int/en/ Section10/Section332/Section1985_9804.htm

9. Gubler DJ, Kuno G, Markoff L. Flaviviruses. In Fields Virology, Knipe DM, Howley PM. 5th ed. Philadelphia: Lippincott Williams \& Wilkins; 2007. p.1155-252.

10. Franco L, Di Caro A, Carletti F, Vapalahti O, Renaudat C, Zeller $\mathrm{H}$, et al. Recent expansion of dengue virus serotype 3 in West Africa. Euro Surveill. 2010;15(7):pii=19490. Available from: http://www.eurosurveillance.org/ViewArticle. aspx?Articleld $=19490$

11. Sang RC. Dengue in Africa. Nairobi: Arbovirology/Viral Haemorrhagic Fever Laboratory, Centre for Virus Research, Kenya Medical Research Institute; 2007. [Accessed 29 August 2010]. Available from: http://www.tropika.net/review/061001Dengue_in_Africa/article.pdf

12. Tamura K, Dudley J, Nei M, Kumar S. MEGA4: Molecular Evolutionary Genetics Analysis (MEGA) software version 4.0. Mol Biol Evol. 2007;24(8):1596-9. 